\title{
Well-being and harm reduction, the consolidated reality of electronic ciga- rettes ten years later from this emerging phenomenon: A narrative review
}

\author{
Pasquale Caponnetto, ${ }^{1-4}$ \\ ${ }^{1}$ Faculty of Health Sciences and Sport, University of Stirling, UK; ${ }^{2}$ Centro per la Prevenzione e \\ Cura del Tabagismo (CPCT), Azienda Ospedaliero-Universitaria Policlinico "G. Rodolico-S.Marco", \\ University of Catania; ${ }^{3}$ Department of Educational Sciences, University of Catania; ${ }^{4}$ Center of \\ Excellence for the Acceleration of Harm Reduction (COEHAR), University of Catania
}

\begin{abstract}
Tobacco use is the greatest threat to public health worldwide, killing more than seven million people annually. This paper, about 10 years after the first review on electronic cigarettes, analyses the evolution that this tool has had in these years. It concludes with comments on the significance of the research and why it constitutes an original contribution. We searched PubMed (National Library of Medicine), and PsycINFO (Ovid) (2006-2020) for studies on e-cigarettes (harms and benefits, e-cigarette use, craving and smoking cessation) and smoking cessation treatment (smoking cessation treatment or varenicline or tobacco cessation or reduction or bupropion or NRT or behavioral treatment or ecigarette) and evidence suggests that they may effective as smoking cessation tool and may be less harmful alternatives to combustible cigarette smoking. Consequently, e-cigarettes could be considered as an applicable instrument for Tobacco Harm Reduction (THR) and smoking cessation.
\end{abstract}

Correspondence: Pasquale Caponnetto, Department of Educational Sciences, University of Catania, Palazzo Ingrassia Via Biblioteca, 4, 95124, Catania, Italy.

E-mail: p.caponnetto@unict.it

Key words: Smoking; e-cigarette; smoking cessation; smoking reduction; harm reduction.

Conflict of interest: PC is paid by the University of Catania as an external part time researcher and adjunct professor of clinical, addiction and general psychology. He has been affiliated to the CoEHAR since December 2019 in a pro bono role. He is co-author of a protocol paper supported by an Investigator-Initiated Study award program established by Philip Morris International in 2017.

Received for publication: 18 November 2020.

Accepted for publication: 24 December 2020.

This work is licensed under a Creative Commons AttributionNonCommercial 4.0 International License (CC BY-NC 4.0).

${ }^{(C)}$ Copyright: the Author(s), 2020

Licensee PAGEPress, Italy

Health Psychology Research 2020; 8:9463

doi:10.4081/hpr.2020.9463

\section{Introduction}

Amongst the World Health Organization (WHO) regions, in 2015 , over 1.1 billion people smoked tobacco. Far more males (36.1\%) than females $(6.8 \%)$ smoked tobacco. Although it is declining worldwide and in many countries, the prevalence of tobacco smoking appears to be increasing in the WHO Eastern Mediterranean Region and the African Region. Amongst the WHO regions Europe has the highest prevalence of tobacco smoking amongst adults (28\%) (WHO, 2018). In Italy, adult smoking prevalence has consistently decreased since recording started in 1957 (Gallus, Lugo, Colombo, Pacifici, La Vecchia, 2013). From 2013 to 2014, Lugo et al. (2015) found an overall smoking prevalence amongst Italian adults of $21 \%$ (26\% of men and $17 \%$ of women). This agrees with recent data from a large household survey (based on 60,000 families and 130,000 individuals) conducted in 2013 by the National Institute of Statistics (ISTAT), showing an adult smoking rate of $21 \%$ (26\% in men and $16 \%$ in women) (ISTAT, 2015). Another study confirmed the decreasing trend observed over the last five decades in men and over the last two decades in women (Gallus et al., 2013). However, no significant decrease in terms of smoking prevalence has been observed in men or women between 2007 and 2014. This likely reflects the lack of adoption of effective and relevant additional antismoking measures after the successful ban introduced in 2005 (Joossens \& Raw, 2014) and smoking prevalence has levelled off over the last few years (Osservatorio Fumo, Droga e Alcol, 2016). In 2005 the Italian government banned smoking in all indoor public places, including offices, cafes, restaurants (except for a few with separate and regulated smoking areas), airports, and railway stations. Italy was the first large country in Europe to introduce a comprehensive smoking ban, which resulted in a further acceleration of the decreasing trend of smoking prevalence in both sexes (Gallus et al., 2006; ISTAT, 2015; Tramacere et al., 2009). In February 2016, the Italian government established new legislation regarding tobacco smoking. New anti-smoking laws have imposed large fines for several offences and make it illegal to smoke in a car carrying children or pregnant women. The laws require cigarette packs to carry health warnings about the effects of smoking. Smoking is also prohibited outdoors near schools and hospitals. Tobacconists caught selling cigarettes to minors risk heavy fines and losing their license. Throwing cigarette butts on the pavement could cost an offender up to 300 Euros (OSSFAD, 2016). However, after the positive initial effects of the law of 2005, the prevalence of smokers in Italy has not decreased further and has instead remained static (OSSFAD, 2018).

Smoking traditional cigarettes is one of the largest risk factors for premature mortality from non-communicable diseases in the 
general population (Stringhini et al., 2017). Tobacco use is the greatest threat to public health worldwide, killing more than seven million people each year (WHO, 2017). Cigarette smoking is the single most preventable cause of death and disease. Smoking-related death is principally caused by lung and other cancers, ischemic heart disease and Chronic Obstructive Pulmonary Disease (COPD) (WHO, 2012; Doll, Peto, Boreham, \& Sutherland, 2004). The risk of serious disease has been shown to diminish rapidly after smoking cessation - 'quitting' - and permanent abstinence markedly reduces the risk of lung cancer and other cancers, ischemic heart disease and COPD. Considering that the main types of these noncommunicable diseases are Cardiovascular Diseases (CVDs), cancers and chronic respiratory diseases, helping traditional cigarette smokers quit and remain abstinent is one of the most effective ways we can improve the public health of Italy, UK and the rest of the world. The associations between traditional cigarette smoking and hazards to physical health are described below. Cigarette smoking is probably the most complex and the least understood amongst the risk factors for CVDs. Cigarette smoke contains several thousand chemicals, though there isn't concordance about the exact number of chemicals. Two studies report traditional cigarettes contain $\approx 4000$ different chemicals with sizes ranging from atoms to particulate matter (Burns, 1991; Zemann, 2011) and other two more recent papers demonstrate tobacco smoke contains more than 7000 chemicals (Perfetti \& Rodgman, 2013; Tobacco Atlas, 2015). Individual smoking behavior, intensity of smoking and the brand of cigarettes smoked further modulate the amount, number, and type of chemicals in tobacco smoke to which an individual is exposed (Conrad, 2011). Importantly, it is likely that it is not just a single compound or a compound class, such as oxidants, but rather a highly complex and changing mixture of compounds that is responsible for disease initiation, progression, and cardiovascular outcome. The interplay of these compounds with the individual's genetic background and the environment defines the onset, location, and pace of CVDs. For the past few decades, it has been clear that smoking is an important (and modifiable) risk factor for CVDs; according to WHO data, smoking is responsible for $10 \%$ of all CVD cases (WHO, 2012). However, for a long time it remained unclear how smoking causes CVDs. In 1993, Celermajer et al. published a study showing that smoking reduces FlowMediated Dilatation (FMD) in systemic arteries in healthy young adults. Smoking not only plays a strong role in CVD initiation, but also significantly contributes to and causes disease progression and fatal cardiovascular outcomes. Current data clearly show that second-hand smoking can also trigger life-threatening conditions. Management and prevention of CVDs is a public health priority, and a simple intervention such as smoking cessation could lead to reduced prevalence of cardiovascular risk factors and, therefore, CVD itself.

There is a clear link between smoking initiation and cancer in later life. Smoking causes more than $48 \%$ of deaths from the 12 types of cancer caused by smoking. Smoking causes more than $80 \%$ of lung cancer deaths as well as $77 \%$ of larynx cancer deaths (Siegel et al., 2015). Approximately 168,000 people in the United States are estimated to die of cancer due to smoking each year. Continued progress in reducing cancer mortality will require more comprehensive tobacco control, including targeted cessation support (de Marco et al., 2004; Siegel et al., 2015). In Europe, the incidence of lung cancer ranges from eight to 62 per 100,000 persons, while prevalence ranges from 26 to 242 per 100,000 persons (Jimenez-Ruiz et al., 2011). In Europe, death rates from lung cancer increased by $58 \%$ between 1960 and 1988, but they declined by $14 \%$ in 1998 , mainly due to the decreased incidence and mortality in males (Ezzati \& Lopez, 2003). The risk of developing lung cancer seems to be affected by the duration of smoking and the number of cigarettes (or cigars, or pipes) smoked daily (La Vecchia Franceschi, \& Levi, 2003). The Relative Risk (RR) ratio between the occurrence of lung cancer amongst smokers and nonsmokers is 15 overall and 25 for heavy smokers (Doll et al., 2004). Reducing the intake of traditional cigarettes smoked per day may support a harm reduction approach but the US Cancer Prevention Study II has shown that the number of years of tobacco smoking is far more critical in predicting lung cancer risk than the number of cigarettes smoked daily (Alberg \& Samet, 2003). The age of starting smoking increases lung cancer risk (Flanders, Lally, Zhu, Henley, \& Thun, 2003); hence, promoting interventions to avoid smoking initiation (typically during the adolescence) are important.

Smoking is also the main cause of many respiratory diseases (CDC, 2004). COPD is predicted to become the third leading cause of death in 2030 (WHO, 2008). Cigarette smoking is the most important risk factor for COPD and it can also promote the onset of exacerbations (Wedzicha \& Donaldson, 2003). Data collected in European countries show that self-reported diagnosis of chronic bronchitis/emphysema (Viegi et al., 1999) or spirometry signs of airflow obstruction (Lundback et al., 2003) are more frequent in smokers than nonsmokers. The risk of developing COPD may be increased not only by the average daily number of cigarettes smoked but even more by cumulative pack-years (Viegi et al., 1999). Moreover, a study has shown that smokers with COPD have higher tobacco consumption, higher $\mathrm{CO}$ levels in exhaled air and higher dependence on nicotine than healthy smokers (Lundback et al., 2003). However, despite COPD being considered as a smoking-related lung disorder, not all traditional cigarette smokers develop this disease. Faner et al. (2014) showed that some smokers may develop irreversible lung obstruction, which is linked with their epigenetic and genetic background (DeMeo et al., 2004) but smoking cessation plays a central role in COPD avoidance. Cigarette smoking is also a risk factor for male and female sexual and reproductive dysfunctions. In particular, it is a risk factor for the onset of erectile dysfunction and traditional cigarettes contain elements that exert a direct harmful effect on male and female germ cells and embryos (Zenzes, 2000). Tobacco use amongst women during their reproductive years is especially dangerous because of the potential for multi-generational harm. Smoking is associated with poorer pregnancy outcomes including infertility, ectopic pregnancy, increased rates of spontaneous abortion and still births. Smoking contributes to preterm birth, low birth weight, increased rates of infant chronic lung disease, wheezing, and an increased risk for Sudden Infant Death Syndrome (DiFranza \& Lew, 1996).

\section{Smoking cessation treatments}

Offering help to quit tobacco use in people dependent on tobacco is one of the six proven policies identified by the WHO Framework Convention on Tobacco Control (FCTC) to address the tobacco epidemic (WHO 2003; CDC, 2004). Key barriers to developing national smoking cessation policies and tobacco-control supportive programmes are: inadequate training and lack of motivation amongst healthcare providers to undertake and deliver smoking cessation activities; lack of resources and government funding; unavailability and inaccessibility of pharmacotherapy products; the absence of mechanisms for financing or subsidising pharmacotherapy products by insurance companies; lack of coor- 
dination between various sectors involved in providing smoking cessation interventions; and, more importantly, the lack of integration of smoking cessation interventions into an overall policy on tobacco control. The FCTC came into force on February 27, 2005 and requires Parties to implement evidence-based measures to reduce tobacco use and exposure to tobacco smoke (WHO, 2013). Article 14 of the FCTC requires Parties to take effective measures to promote cessation and adequately treat tobacco dependence. Guidelines for the implementation of Article 14 recommend establishing specialized tobacco dependence treatment services, making medications widely available and considering emerging research evidence and novel approaches to cessation. The 2008 US Guide to Quitting Smoking recommends that, except for groups with contraindications or for whom smoking cessation drugs have uncertain efficacy (e.g., users of smokeless tobacco, light smokers, pregnant women, nursing women, and teenagers), clinicians should encourage all smokers intending to quit smoking to take smoking cessation medications combined with smoking cessation advice (2008 PHS Guideline Update Panel, Liaisons, and Staff, 2008)

There is evidence regarding the efficacy of the drugs used in smoking cessation (Bauld, Bell, McCullough, Richardson, \& Greaves, 2010; Cahill, Stevens, Perera, \& Lancaster, 2013). The drugs are most effective when used incombination with behavioral therapies and support. Cognitive and behavioral interventions, such as motivational interviewing and relapse prevention, are an essential adjunct for the efficacy of these treatments (Bauld et al., 2010; Cahill et al., 2013). In the general population there are three principal approved drug therapies, according to US International Guidelines: Nicotine Replacement Therapy (NRT), varenicline, and bupropion (2008 PHS Guideline Update Panel, Liaisons, and Staff,, 2008).

\section{Nicotine replacement therapy}

NRT has an established evidence base in the general population. Multiple formulations are available - gum, lozenges, oral strips, sublingual tablets, inhalers, mouth and nasal sprays, and transdermal patches (16- or 24-hours release). All have comparable efficacy. These different formulations allow for better tailoring to individual requirements including the use of combinations if required. For example, a patient may be prescribed a transdermal nicotine patch and additionally use a nicotine inhaler to supplement blood nicotine concentrations at times of particular craving or risk of relapse (Stead et al., 2012). The OR of abstinence for any form of NRT compared with placebo is 1.84 (Cahil et al., 2013). Combined NRT formulations have been shown to result in higher abstinence rates than single NRT. The OR of abstinence for combination NRT compared with single NRT products is 1.43 (Cahil et al., 2013).

\section{Varenicline}

Varenicline has comparable efficacy with combination NRT but has superior efficacy to nicotine replacement monotherapy. It is therefore recommended as an equal first-line drug for smoking cessation. Varenicline acts as a partial agonist at central nicotinic acetylcholinergic receptors, which are important in mediating the reinforcement associated with tobacco smoking. During treatment, drug binding partially activates these receptors thereby reducing withdrawal symptoms and cravings. If the patient lapses and smokes, varenicline reduces the access of nicotine to the receptors. By limiting nicotine binding, varenicline reduces its rewarding effect. It is recommended that varenicline is started a week or two before the patient quits smoking. This is because a continuous peri- od of dosing is required before sufficient receptors are occupied and optimal drug efficacy is achieved. The recommended dosage is one $\mathrm{mg}$ twice daily (for 12 weeks) following a one week up-titration (2008 PHS Guideline Update Panel, Liaisons, and Staff, 2008). In the most recent Cochrane review, Cahill, LindsonHawley, \& Thomas, (2016) reported that the OR of continuous abstinence for varenicline compared with placebo was 2.24; varenicline was more effective when compared with bupropion (OR 1.39) and single-product NRT (OR 1.25), and was similarly effective compared with combination NRT. Varenicline is principally eliminated by the kidneys so reduced doses (or alternative treatments) are recommended for patients with renal impairment. Common adverse effects include nausea, headache and insomnia.

\section{Bupropion}

Bupropion was originally developed as an antidepressant. It has dopaminergic and adrenergic actions and is an antagonist at the nicotinic acetylcholinergic receptor; however, its precise mode of action in smoking cessation is uncertain. It has equivalent efficacy to nicotine replacement monotherapy but is less effective than varenicline and is therefore considered a second-line option (2008 PHS Guideline Update Panel, Liaisons, and Staff, 2008). The recommended dosage of bupropion is $150 \mathrm{mg}$ twice daily. Several meta-analyses have confirmed the efficacy of bupropion (Hughes, Stead, \& Lancaster 2007; 2008 PHS Guideline Update Panel, Liaisons, and Staff, 2008). In a network meta-analysis by Cahill et al. (2013), the OR of abstinence for bupropion compared with placebo was 1.82. Bupropion was of similar efficacy to single product NRT (RR 0.99) and less effective for quitting compared with varenicline and combination NRT. Bupropion has been shown to decrease nicotine/tobacco withdrawal symptoms and cigarette cravings (Mooney \& Sofuoglu, 2006). Bupropion is contraindicated in patients with a history of seizures. Common adverse effects include difficulty concentrating, insomnia and nightmares. Bupropion undergoes significant hepatic CYP 2B6 metabolism to an active metabolite (hydroxybupropion), which is later excreted renally. Dose reduction is necessary in patients with hepatic or renal disease. There are potential interactions with other drugs metabolized by this system including antipsychotics and selective serotonin reuptake inhibitors.

\section{Tobacco harm reduction}

The history of Tobacco Harm Reduction (THR) may be traced back to at least 1974, with the publication of a special article in the Lancet by British tobacco addiction research expert Michael A.H. Russell (1974). In essence, harm reduction as part of a tobacco control strategy involves trying to separate the risk associated with inhaling smoke from that of taking nicotine. As Russell noted 30 years ago, "There is little doubt that if it were not for the nicotine...people would be little more inclined to smoke than they are to blow bubbles or light sparklers" (Russell, 1974). More recently, the National Institute for Health and Care Excellence (NICE) in the UK has described THR as reducing the diseases and deaths caused by smoking traditional cigarettes (NICE, 2011). NICE produced the world's first formal guidelines on THR between 2011 and 2013. These guidelines address reducing harm from smoking traditional cigarettes in order to help smokers, particularly those who are highly dependent on nicotine, and may not be able (or do not want) to stop smoking in one step; may want to stop smoking, without necessarily giving up nicotine; and may not be ready to stop smoking, but want to reduce the amount that they smoke 
(NICE, 2013). Despite the NICE harm reduction guidelines, approved smoking cessation treatment in the UK and other countries normally requires nicotine addicted smokers to abstain from tobacco and nicotine entirely. Many smokers are unable - or at least unwilling - to achieve this goal, and so they continue smoking in the face of impending adverse health consequences. In effect, established approaches to smoking cessation present smokers with just two alternatives: stop smoking or suffer the harmful effects of continuing smoking. However, THR arguably provides a third choice for smokers. It involves the use of alternative sources of nicotine as a replacement for smoking. E-cigarettes as a product category can fit within a THR paradigm. They can deliver nicotine without the combustion products that are responsible for nearly all of smoking's damaging effects. These products and key available evidence about their use is outlined in the next sections of this chapter.

\section{The E-cigarettes}

E-cigarettes are a part of a series of emerging products often referred to as Alternative Nicotine Delivery Systems (ANDS) or Electronic Nicotine Delivery Systems (ENDS). The term ENDS was created by the WHO's Study Group on Tobacco Regulation in 2009 to classify a collection of battery-powered devices that provide nicotine flavourings and other additives to the user in aerosol form. A first version of an e-cigarette was patented as early as 1965 in the USA by Gilbert (Gilbert, 1965). The Gilbert e-cigarette resembled a traditional cigarette but instead of burnt tobacco, it allowed the user to draw warm flavoured vapour into the mouth or lungs. A cartridge held a chemical solution and an insulated tube or light bulb powered by a battery provided a heating element to heat the solution. However, despite this innovative idea, Gilbert was never able to bring this product successfully to market. Some years later, in 1986, the 'Favor' cigarette was developed as a non-combustible nicotine-containing product that also resembled a cigarette in appearance (Ling \& Glantz, 2005). The device was made of a plastic tube containing a paper soaked with nicotine in order to simulate the traditional cigarette effect without vapour creation. The Food and Drug Administration (FDA) in the USA deemed this type of device to be a nicotine delivery system and as consequence classified Favor as a drug and banned it (Sleight, 2016). Following these very early designs, the modern e-cigarette was developed in 2003 by a Chinese pharmacist, Hon Lik. He created an electronic atomizing cigarette for smoking cessation after his father (a smoker) died of lung cancer. This e-cigarette was composed of a batteryoperated device designed to vaporize a liquid solution of propylene glycol and vegetable glycerine in which nicotine was dissolved. No tobacco was used in the device. Puffing activated a batteryoperated heating element in the atomizer and the liquid in the cartridge was vaporized as a plume of a dense mist and inhaled (Hon, 2003). This e-cigarette prototype showed promise, and as a result in 2004 it was introduced on the Chinese market under the company name Ruyan. From there many other companies began to develop similar devices building on this initial prototype. The technology and range of products rapidly evolved and the market grew considerably from 2010 onwards. These products have now spread rapidly across Europe, the USA and a number of other areas and countries (Sanford, Goebel, \& Lynne, 2014).

\section{E-cigarette market and types}

As of 2014, it was estimated that over 460 e-cigarette brands were available on the global market (Zhu et al., 2014). However, despite the potential product differences, some characteristics of ecigarettes appear to be consistent across products. These include: a cartridge containing propylene glycol or glycerine mixed with different nicotine concentrations and a battery powered heating component which transforms the liquid substance into an aerosol form when air is drawn through the device (Cobb, Byron, Abrams, \& Shields, 2010). E-cigarettes are also sold as either disposable or reusable, refillable products. E-cigarette devices vary from first generation, second generation, and third and fourth generation products.

First-generation e-cigarettes appear similar to traditional cigarettes, usually with a white body made of plastic and a tan mouthpiece (other first-generation e-cigarettes were slightly longer or narrower than a traditional cigarette and were black or coloured). These devices are described as "cigalikes." First generation models included a cartridge designed for the part of the device that holds the e-liquid, which is either prefilled with the liquid or ready to be filled. The user then connects the cartridge to the heating element and atomizer that are themselves connected to the battery, and the "cigalike" is ready for use (Zhu et al., 2014).

Second-generation e-cigarettes include devices that resemble fountain pens, are large and cylindrical, and are often described as e-cigarettes with "tank systems" in consideration of the transparent reservoir that holds larger amounts of e-liquid (about two or three $\mathrm{ml}$ ) than previous first generation "cigalike" models.

Third generation devices are a diverse product category, and they appear totally different from traditional cigarettes in their appearance, principally because many are square or rectangular and customizable by changing batteries and atomizers. Since the beginning of the availability of e-cigarettes' component elements, users have been modifying their own e-cigarettes by building their own customized e-cigarettes, which are called "mods." Users can adjust the battery voltage and combine the e-liquid, choosing different flavours and nicotine levels (Richtel, 2014; Lee \& Kim, 2015).

Fourth generation devices are the most advanced and powerful and differ from third-generation e-cigarettes in the following small details: they enable control over the temperature of the heating coil and can be used at much higher power levels (e.g., >200 W) compared with most earlier e-cigarettes (Strongin, 2019).

E-cigarette devices have evolved substantially over time, from early-generation cigalike e-cigarettes to more advanced modifiable tank-style versions. One of the latest products to be introduced on the market, initially in the USA, is the JUUL e-cigarette (Truth Initiative, 2018). JUUL is a non-modifiable compacted closed system e-cigarette and represents one of a newer generation of pod devices.

JUUL has two basic components: the device, which includes the battery and temperature regulation system, and the prefilled eliquid cartridge, called a 'pod', that comes in a variety of flavours (tobacco, mango, mint, and others). The original JUUL device pods contained $0.7 \mathrm{~mL}$ of e-liquid with $5 \%$ nicotine by weight, although lower strength nicotine options are now on the market, particularly in Europe.

The JUUL pods also serve as the mouthpiece for the product. JUUL is rechargeable by a USB port and is rectangular and small in size, fashioned to look like a computer flash drive (Kee, 2018). The characteristics that make JUUL different from its predecessors 
include its design (called the 'iPhone of e-cigarettes') (Radding, $2015)$, its high levels of nicotine $(0.7 \mathrm{~mL}$ or $59 \mathrm{mg} / \mathrm{mL}$ per pod) and the use of a specific e-liquid formula, JUUL salts, based on the nicotine salts found in leaf-based tobacco rather than free-based nicotine. JUUL provides a nicotine concentration comparable with a traditional cigarette, with the nicotine peaking in about five minutes, and delivers nicotine 1.25-2.7 times faster than competing ecigarettes on the market (Brown \& Xing, 2015; JUUL, 2018; Lawler, 2018).

\section{Existing evidence on e-cigarette harms and benefits}

Although e-cigarettes have only become widely used in the last few years, research evidence on these devices has been growing rapidly. This evidence is not always consistent and sometimes contradictory, but there is a growing consensus that these products are significantly less harmful than traditional cigarettes (Farsalinos \& Polosa 2014). That said, important research questions remain regarding any potential harms from use and also the potential benefits of use. These questions include, for example, whether these products are effective aids for smoking cessation, promote uptake by nontobacco users, sustain nicotine dependency via dual use, slow intentions to quit in dual users, or encourage relapse to cigarette use amongst former smokers (Glasser et al., 2017). Ecigarette use is a complex and dynamically evolving behaviour. To advance knowledge of the impact of e-cigarettes use on smoking status, it will be necessary to conduct prospective studies considering relevant descriptors of vaping behaviour such as frequency of use (e.g. focusing on daily users, and not just on those who are experimenting), reasons for using e-cigarettes (e.g. to quit smoking vs. out of curiosity), and product design (e.g. closed vs. open systems, nicotine containing vs non-nicotine containing products, etc.). Reasons for vaping, the type of device and e-liquid, frequency of use, and the accompanying sensory and craving-control experiences may have some impact on smoking behaviours (Polosa et al., 2017). E-cigarettes have ingredients that are not inert and are likely to have some potential health risks. While traditional cigarette combustion generates toxic substances correlated with cancers, respiratory disorders and CVDs, e-cigarettes usage delivers potentially toxic substances involving fine particulate matter, metals that are known to probably determine adverse health effects related to cancers, respiratory disorders and CVDs.

E-cigarette-related toxicants and carcinogens: A number of studies have tried to assess the RRs of e-cigarette use compared with tobacco smoking, in particular, the extent to which there is any reduction in exposure to harmful toxicants as compared with smoking traditional cigarettes. For example, in one study, a research group provided 40 smokers with e-cigarettes in a choice of eight flavours with $12 \mathrm{mg}$ or $24 \mathrm{mg}$ of nicotine. The researchers collected urinary cotinine, the tobacco-specific carcinogen 4(methylnitrosamino)-1-(3-pyridyl)-1-butanol (NNAL), and eight volatile organic compounds at BL visit and a four week follow-up visit. They observed that $\mathrm{CO}(\mathrm{p}<0.001)$, NNAL $(\mathrm{p}<0.01)$, and metabolites of benzene $(p<0.01)$ and acrylonitrile $(p=0.001)$ decreased significantly in smokers who switched to e-cigarettes (Pulvers et al., 2016). Another recent study (Shahab et al., 2017) compared, in 37 participants of traditional cigarette-only smokers, and in 72 smokers and 72 ex-smokers with long-term e-cigarette use or with use of NRT, the exposure to nicotine, tobacco-related carcinogens and toxicants. Urine and saliva samples were collected and there were no differences in salivary or urinary biomarkers of nicotine intake after controlling for confounders. The study found lower carcinogen and toxicant levels in participants who had switched completely from smoking to either exclusively ecigarettes or NRT use. However, both of the studies, conducted by Pulvers et al. and Shahab et al., used samples too small to assess the potential association of different types of e-cigarettes, did not assess indirect exposure and were limited by the number of biomarkers available. A large study used data from 5,105 of the U.S. adults participating in the Population Assessment of Tobacco and Health (PATH) study and compared exposure to toxicants amongst e-cigarettes users, non-smokers and smokers. This study examined urine samples for key biomarkers of exposure to harmful chemicals, Tobacco-Specific Nitrosamines (TSNAs), metals, Polycyclic Aromatic Hydrocarbons (PAHs), and volatile organic compounds (VOCs), and suggested possible benefits for smokers who totally switched to e-cigarettes. Exclusive users of e-cigarettes were exposed to more toxicants than people who did not use any form of traditional cigarette, but at significantly lower levels than smokers (Goniewicz et al., 2018).

\section{E-cigarette use and cancer risk}

The cancer risk related to e-cigarette usage would be expected to be less than traditional cigarettes based on the fact that ecigarettes include nicotine but not many of the other toxicants in traditional combusted cigarettes. On this basis their use should result in a reduced burden of carcinogens compared with smoking (Chen et al., 2017; Stephens, 2018).

A small number of studies to date have focused on e-cigarette users and cancer. For example, in a study conducted by Franco, Trapasso, Puzzo, and Allegra (2016), oral cells were collected by scraping the oral mucosa from a population of 65 participants from three groups: traditional cigarette smokers $(n=23)$; e-cigarette users $(n=22)$; and non-smokers or users of traditional cigarettes and e-cigarettes $(n=20)$. Their findings showed that compared with non-smokers of traditional cigarettes and non-users of e-cigarettes, the mean number of micronucleated cells/ 1,000 cells and the measure of total micronuclei/1,000 cells was respectively $160 \%$ and $633 \%$ higher in traditional cigarette smokers and $21 \%$ and $133 \%$ higher in e-cigarette users. This study, despite not presenting any evidence on the reliability of the micronucleus assay, nor reporting all important p-values, or several potential confounding factors (e.g. age, or age at smoke initiation), showed that the average micronuclei burden was high in e-cigarette users compared with never smokers, and was higher in traditional cigarette smokers compared with e-cigarette users and never smokers. A further study assessing RRs compared with smoking was conducted by Manzoli et al. (2017). They enrolled 932 participants at BL assessment with the following sample sizes at the end of the 24-month follow-up: 363 smokers of only traditional cigarettes throughout follow-up, 97 users of only e-cigarettes throughout follow-up, and 37 dual users of both traditional and e-cigarettes. This study reported the definition "any cancer" as one of the possible Serious Adverse Events (SAEs) in participants with this distribution: 0.8\% $(3 / 363)$ in traditional cigarettes users, 2.1\% (2/97) in e-cigarettes users and $0 \%(0 / 37)$ in dual users. The risk ratios calculated from these data, using traditional cigarettes as the referent category, were 2.49 (95\% CI $0.42-14.72)$ for e-cigarettes only and 0 (95\% CI not estimable) for dual use. Considering that all participants were previous traditional cigarette users, the findings did not provide any indication of elevated cancer risk from sole use of e-cigarettes. However, the study did have limitations including: the absence of consideration of traditional cigarette smoking history, a small sample size to evaluate the endpoint of cancer, and self-reported cancer 
data. Studies in humans focusing on cancer and e-cigarette use are relatively few in number, and to date there are no epidemiological studies on the possible association between e-cigarette use and cancer in humans. This makes it difficult to draw definitive conclusions about any association between e-cigarette use and risk of cancer in human populations. However, a joint statement on ecigarettes by Public Health England and other UK public health organisations (PHE, 2016) developed a shared agreement document stating that e-cigarettes are significantly less harmful than smoking. A recent study conducted by Stephens (2018) measured emissions from cigarettes and e-cigarettes, calculating lifetime cancer risk using daily consumption estimates, and calculated that e-cigarette cancer potencies were largely found to be only a small fraction of those of smoking $(0.4 \%)$.

A different body of research has focused on e-cigarettes' effects on users of traditional cigarettes with or without pre-existing respiratory conditions such as COPD or asthma. These studies in humans examine the effect of switching to e-cigarettes (single or dual use), examine health effects of e-cigarettes compared with traditional cigarette usage, and overall suggest that smokers with preexisting respiratory conditions such as asthma and COPD may experience some benefits from switching to e-cigarettes (Campagna et al., 2016; Cibella et al., 2016; Polosa et al., 2014 a, b; Polosa et al., 2016 a, b). Our research group in Italy has contributed to this literature on respiratory health and e-cigarettes. In a one-year RCT of healthy smokers who abstained from cigarette smoking and switched to e-cigarette use, we observed improvements in their exhaled breath measurements, including fractional nitric oxide concentration in exhaled breath (FeNO) and exhaled carbon monoxide (eCO) (Campagna et al., 2016). A 24-month prospective study (Polosa et al., 2016) demonstrated improved respiratory symptoms and lung function in 16 former smokers who switched to e-cigarettes, suggesting that e-cigarette use could potentially contribute to reversing the harm from combustible tobacco in smokers with asthma. Polosa et al. (2017) conducted a study with young-adult never-smoking, daily e-cigarette users who were followed up for at least $3 \frac{1}{2}$ years by our research group. No worsening in spirometric indices (i.e. lung function), no development of respiratory symptoms, no changes in markers of lung inflammation in exhaled air, and no signs of early lung damage on High Resolution Computed Tomography (HRCT) were detected. However, a limitation of these studies is that they were conducted in a small number of smokers selected retrospectively from the same region and primarily from a single study research group, which limits generalizability of the results. Different findings come from a high-quality RCT with large sample size, where Cravo et al. (2016) reported no difference in lung function in smokers who switched to e-cigarettes. In this study smokers with respiratory conditions were excluded and two cohorts of smokers were randomised to either change to e-cigarettes with nicotine or continue smoking traditional cigarettes. The authors reported no significant positive or negative changes in pulmonary function tests after 12 weeks between the two groups (Cravo et al., 2016). Several recent studies have evaluated acute cardiovascular effects such as modifications in Blood Pressure (BP) levels and Heart Rate (HR) following e-cigarette use. Studies investigated modifications in BP levels after e-cigarette use (Cooke, Pokhrel, Dowling, Fogt, \& Rickards, 2015; Farsalinos, Spyrou, Tsimopoulou, Stefopoulos, Romagna, \& Voudris, 2014; Fogt, Levi, Rickards, Stelly, \& Cooke, 2016; Moheimani et al., 2017; Szołtysek-Bołdys et al., 2014; Yan \& D'Ruiz, 2015). These studies had some inconsistent results, with the majority finding weak positive increases or no modifications and harms using e-cigarettes.
Previous studies, using first and second generation e-cigarettes, found no changes in HR following e-cigarette use (Farsalinos et al., 2014; Szołtysek-Bołdys et al., 2014; Vansickel, Cobb, Weaver, $\&$ Eissenberg, 2010) but these were conducted with devices characterized by slight or no increase in blood nicotine levels. In one prospective study, using first and second generation e-cigarettes and conducted by our team in Italy, systolic BP was significantly reduced at week 52 compared with BL (132.4 +/- 12.0 vs. $141.2+/$ $10.5 \mathrm{mmHg}, \mathrm{p}<0.00)$ amongst participants who had reduced smoking ( $>50 \%$ reduction) and in those who had quit smoking with the use of e-cigarettes. These findings suggest that e-cigarette use does not elevate BP (Farsalinos et al., 2016). This study did not find any changes in BP when assessing all participants, because the vast majority initially had normal BP and no change in this parameter was expected to occur within the 12-month duration of the survey. The interest was directed towards the population with an initially high BP (high-normal or higher, as defined by the European Society of Cardiology). The reduction in BP was evident even after adjusting for confounders such as age, gender and weight gain. However, these early studies may not reflect the effects of newer devices. Some more recent research using third generation devices, which increase blood nicotine levels, has identified some increase in HR just after e-cigarette use (Cooke et al., 2015; Spindle et al., 2016; St. Helen, Dempsey, Havel, Jacob III, P., \& Benowitz, 2017). A key factor to consider when drawing any conclusions about potential health risks from e-cigarettes is the extent to which studies directly compare vaping with tobacco smoking. Those studies that have aimed to directly compare the two have fairly consistently found reduced levels of harm if participants switch completely to vaping from smoking. Looking across the body of literature, there is convincing evidence that vaping amongst exsmokers reduces exposure to toxicants that are carcinogenic and may increase the risk of cancer. In addition, there is now good evidence that risks to both cardiovascular (Yan \& D'Ruiz, 2015; D'Ruiz, O'Connell, Graff, \& Yan, 2017) and respiratory health (Polosa et al., 2014a,b; 2016b,c) are reduced when smokers switch from using combustible tobacco to vaping.

\section{E-cigarette use, craving and smoking cessation}

Smokers with considerable histories of cigarette usage report using e-cigarettes to alleviate nicotine withdrawal generated by smoking cessation or to satisfy cravings for these traditional cigarettes (Etter \& Bullen, 2014). An RCT (Adriaens, Van Gucht, Declerck, \& Baeyens, 2014) on smoking behaviour and use ecigarettes in 48 traditional cigarettes smokers randomised participants into two e-cigarette groups and one control group.

One of the researchers' objectives was to assess whether ecigarettes decreased craving in the short term, and during the laboratory studies in the first two-month period of the study, researchers assessed craving and found that e-cigarettes proved to be just as effective in suppressing the craving for smoking as traditional tobacco cigarettes. Dawkins and Corcoran (2014) enrolled 14 experienced vapers and asked them to abstain overnight from their traditional cigarettes. In the morning, they were presented with a first-generation e-cigarette with $18 \mathrm{mg} / \mathrm{mL}$ nicotine, from which they were invited to take 10 puffs. As a result, the nicotine craving and urge to smoke were significantly reduced in these participants. In a second study, Dawkins, Munafo, Christoforou, Olumegbon, and Soar, (2016) presented 63 abstinent smokers who were not current e-cigarette users with either a red or a white first 
generation e-cigarette containing $18 \mathrm{mg} / \mathrm{mL}$ of nicotine in the form of "tobacco" flavored e-liquid after a 10-hour abstinence period. Their results suggest that the visual appearance of an e-cigarette has an effect on cigarette craving reduction. These researchers found that the more the e-cigarette resembled a traditional cigarette, the stronger the craving reduction. Another study focused on e-cigarettes' flavour (Goldenson et al., 2016) and showed that nicotine-free and nicotine-containing e-cigarettes produced greater appeal when containing sweet flavours than when containing non-sweet flavours or no flavour.

Smoking is a difficult addiction to break and many smokers persist in tobacco use for numerous years, typically cycling through multiple periods of remission and relapse (Caponnetto, Keller, Bruno, \& Polosa, 2013; Caponnetto, Russo, et al., 2013). Yet, while complete cessation of any nicotine use may be the most desirable final outcome, substitution of traditional cigarettes with alternative non-combusted forms of nicotine delivery, such as ecigarettes, is now a relatively new option available to smokers. Surveys of e-cigarette users have examined reasons for use and, overall, these suggest that users report using them to help quit smoking, to reduce cigarette consumption, to relieve tobacco withdrawal symptoms, and to continue some of the behavioural aspects of smoking with perceived reduced risks to (Etter, 2010).

The first RCT examining the impact of e-cigarettes on smoking behaviour was conducted by the research team at the Centro per la Prevenzione e Cura del Tabagismo-University of Catania (CPCT), Italy, led by Professor Riccardo Polosa and including the $\mathrm{PhD}$ candidate. This study found that smokers not immediately willing to quit who used e-cigarettes substantially decreased daily cigarette consumption without significant side effects (Caponnetto, Campagna, et al., 2013). The trial involved 300 smokers without mental health conditions recruited from advertisements in a local newspaper in Catania. This double blind RCT examined the effects of using a $7.2 \mathrm{mg}$ nicotine e-cigarette, hereafter referred to as Group A; $7.2 \mathrm{mg}$ for six weeks, then transition to $5.4 \mathrm{mg}$ nicotine e-cigarettes, hereafter referred to as Group B; and nicotine-free ecigarettes, hereafter referred to as Group $\mathrm{C}$, on smoking reduction/cessation and adverse effects. The primary outcome of the study was $>50 \%$ reduction in Cigarettes Per Day (CPD) at the 52week study visit from BL. The secondary outcome was sustained smoking abstinence at the 52-week study visit.

The eligibility for study inclusion was adult smokers in good health, age 18-70 years, using $\geq 10$ factory-made CPD for at least the past five years, not attempting/wishing to quit in the next 30 days. Exclusion criteria for the study were symptomatic CVD, symptomatic respiratory disease, regular psychotropic medication use, current or past history of alcohol abuse, use of smokeless tobacco or NRT, pregnancy or breastfeeding. Participants were not encouraged or given any motivation to cease smoking. Study participants were instructed to use the product ad libitum throughout the day, not to exceed a four cartridge/day maximum as recommended by the manufacturer of the product. Participants attended follow-up visits at 2, 4, 6, 8, 10, 12, 24, and 52 weeks. At each of these visits, participant eCO levels were recorded, study diaries were given to study personnel, and unused study products were turned in. After 12 weeks, no additional cartridges were provided to the participants. However, participants were told they could continue to use the e-cigarettes. Saliva cotinine levels were measured at six and 12 weeks in participants who reported no smoking and had an eCO $\leq$ seven ppm.

Loss to follow-up was 35/100 (35\%) in Group A, 37/100 (37\%) in Group B, and 45/100 (45\%) in Group C. All patients were analysed in the group to which they were first allocated with an intention to treat philosophy. At week 52, 10/100 (10\%) of those allocated to Group A, 9/100 (9\%) of those allocated to Group B and $12 / 100(12 \%)$ of those allocated to Group $C$ had reduced their CPD by $\geq 50 \%, p=0.24$. At week $52,13 / 100$ (13\%) of those allocated to Group A, 9/100 (9\%) of those allocated to Group B and 4/100 (4\%) of those allocated to Group C had achieved smoking abstinence and had eCO concentrations of $\leq$ seven ppm, $\mathrm{p}=0.24$. Self-reported adverse effects amongst the remaining 183 participants of the study at 52 weeks included: throat irritation 37/183 (20.2\%), mouth irritation 34/183 (18.6), dry cough $37 / 183$ $(20.2 \%)$, headache $5 / 183(2.7 \%)$, shortness of breath $15 / 183$ $(8.1 \%)$. No SAEs (that is, major depression, abnormal behaviour or any event requiring an unscheduled visit to the family practitioner or hospitalization) occurred during the study.

The second RCT examining the impact of e-cigarette use on smoking behaviour was conducted by the research team at the National Institute for Health Innovation, University of Auckland and Health New Zealand, Christchurch, led by Professor Chris Bullen (Bullen et al., 2013). This research evaluated the quitting efficacy, acceptability, and adverse effects of e-cigarettes, comparing active (16 mg nicotine) e-cigarettes with nicotine patch and placebo ( $0 \mathrm{mg}$ nicotine) e-cigarettes. The trial was a three-arm parallel group RCT. A total of 657 smokers were randomised into one of three groups: a group who used active e-cigarettes for 12 weeks after quitting, a group using nicotine patches for 12 weeks, or a group using placebo e-cigarettes for 12 weeks. Quit rates were assessed at three and six months after the quit date. The primary outcome was the proportion of smokers who maintained sustained continuous abstinence from smoking for six months after their quit day. Secondary outcomes were: continuous abstinence at one and three months, seven-day point prevalence, proportion of participants who significantly reduced daily cigarette smoking by at least $25 \%$ in terms of numbers of CPD, and AEs.

Those eligibility for study inclusion were adult smokers in good health, age $\geq 18$ years, who had smoked at least 10 cigarettes per day for the past year and were motivated to stop smoking. Exclusion criteria for the study were: having had a heart attack, stroke or severe angina in the previous two weeks; poorly controlled asthma or other airway disease from self-report; poorly controlled diabetes mellitus; severe allergies; poorly controlled psychiatric disorders; or current drug dependence other than that involving nicotine. Pregnant and breastfeeding women were excluded.

At six months, the verified continuous abstinence was $7.3 \%$ (21 of 289) with nicotine e-cigarettes, 5.8\% (17 of 295) with nicotine patches, and $4.1 \%$ (three of 73 ) with no-nicotine e-cigarettes. No significant difference between e-cigarette users compared with nicotine patch users was found in six-month abstinence rates (RR $1.26,95 \% \mathrm{CI}=0.68$ to 2.34 ).

A significantly higher proportion of e-cigarettes users, compared with nicotine patch users, achieved a $50 \%$ or more reduction in traditional cigarette use (57\% vs 41\%; RR 1.41, 95\% CI 1.20 to 1.67). The first group reduced traditional cigarette consumption by an average of 9.7 (SE 0.4) CPD, compared with a reduction of 7.7 (SE 0.4$)$ showed by nicotine patch users $(p=0.002)$. The authors noted no evidence of an association between AEs or SAEs and products used in their study. There are also non-randomised studies of e-cigarettes for smoking cessation and this literature is too extensive to summarise those here.

Following these early studies, a large number of subsequent studies have been published focusing on e-cigarettes for smoking cessation. These have been summarized and assessed in a number of systematic reviews; in fact, there are 22 systematic reviews of e- 
cigarettes for smoking cessation published to date.

The most robust of these is the Cochrane review of e-cigarettes for smoking cessation, first published in 2014 and subsequently updated in 2016. In this Cochrane review of the effect of ecigarettes on smoking cessation, Hartmann-Boyce, McRobbie, Bullen, Begh, Stead, and Hajek (2016) identified 24 studies: two RCTs that followed participants for at

least six months (described in section 2.3.1 above) and 22 observational studies which followed participants for less than six months or did not put people into treatment groups so could not directly compare e-cigarettes with something else. The authors also identified 15 ongoing trials. The two RCTs compared ecigarettes with and without nicotine and had a combined sample size of 662 participants (Bullen et al., 2013; Caponnetto, Campagna, et al., 2013). One trial included minimal telephone support, one recruited smokers not intending to quit, and both used early e-cigarette models with low nicotine content and poor battery life. In the meta-analysis of the two trials, e-cigarettes had a higher smoking cessation rate compared with placebo e-cigarettes. Nine per cent of participants using an e-cigarette containing nicotine successfully quit for at least six months compared with $4 \%$ of participants using a placebo e-cigarette (RR 2.29, 95\% CI 1.05 to 4.96). Also, in this review, none of the studies found that smokers who used e-cigarettes short- to mid-term (for two years or less) had an increased health risk compared with smokers who did not use ecigarettes.

Hartmann-Boyce et al. (2016) judged the RCTs to be at low risk of bias, though they rated the overall quality of the evidence as 'low' or 'very low', because of imprecision due to the small number of trials. According to the Grading of Recommendations, Assessment, Development and Evaluations (GRADE) system used in Cochrane reviews, a 'low' grade means that further research is very likely to have an important impact on the confidence in the estimate of effect and is likely to change the estimate. A 'very low' grade means there is uncertainty about the estimate.

The WHO commissioned El Dib et al. (2017) to conduct a systematic review and meta-analysis of the effect of e-cigarettes on traditional cigarette use amongst smokers. This research compared e-cigarettes with nicotine with e-cigarettes without nicotine and two independent reviewers, who also extracted data and assessed the studies' risk of bias, independently evaluated prospective observational studies published up to December 2015. Three eligible randomised trials were considered, with 1,007 participants, but the third randomised trial was excluded because the effect of ecigarettes versus no e-cigarettes couldn't be compared for 24 weeks; hence, a total sample of 481 participants was considered. These researchers also identified nine eligible cohort studies with 13,115 participants but concluded overall that there was still limited evidence about the effectiveness of e-cigarettes for smoking cessation. These two systematic reviews considered a similar group of studies and arrived at similar conclusions: that the available studies are few and that additional evidence is needed to provide a definitive conclusion. However, the reviews differed slightly in their interpretation. The Hartmann-Boyce et al. (2016) systematic review found a statistically significant effect of nicotine ecigarettes compared with e-cigarettes without nicotine and considered this an important effect of e-cigarettes on quitting, and El Dib et al. (2017), using the same studies, were more cautious in their conclusions.

Kalkhoran and Glantz (2016) conducted a systematic review and meta-analysis and identified 38 eligible studies about the impact of e-cigarette use on smoking cessation amongst adult smokers. In this systematic review and meta-analysis, the authors included cohort studies, cross sectional studies, and randomised and non-randomised clinical trials published up to December 2015 and concluded that e-cigarettes are associated with significantly less quitting amongst smokers (Kalkhoran \& Glantz, 2016).

Khoudigian et al. (2016) published a systematic review and meta-analysis of RCTs and observational studies about smoking cessation for at least 24 weeks after the start of e-cigarette adoption. These authors observed that despite their findings, which suggested that e-cigarettes with nicotine increased the proportion of smokers who quit (RR of $2.02(95 \% \mathrm{CI}=0.97-4.21)$, this change was not statistically significant in their meta-analysis. Consequently, the authors concluded that several larger high-quality studies are needed to inform policy decisions.

In conclusion, the existing systematic reviews on e-cigarettes for smoking cessation have inconsistent results. However, all identify the need for further research, in particular further RCTs.

Since the trials, observational studies and systematic reviews mentioned above were conducted, there have been furthermore recent studies on e-cigarettes for smoking cessation. In particular, three recent population-based, cross sectional studies have been conducted that identify promising smoking cessation rates, particularly amongst more frequent e-cigarette users (Giovenco \& Delnevo, 2018; Levy, Yuan, Luo, \& Abrams, 2017; Zhu, Zhuang, Wong, Cummins, \& Tedeschi, 2017).

Firstly, Giovenco \& Delnevo (2018) conducted a retrospective cohort study by including smokers and former smokers who quit in 2010 or later. Amongst these participants daily e-cigarette users and former smokers were $52 \%$, daily e-cigarette users and never ecigarette users were 28\%, [adjusted Prevalence Ratio (aPR) 3.15, $95 \% \mathrm{CI}=2.66,3.73]$. Compared with participants who never used e-cigarettes (aPR $0.38,95 \% \mathrm{CI}=0.32,0.47$ ), those who previously used e-cigarettes but did not use them currently and those who used them on only some days were less likely to be former smokers (aPR 0.67, 95\% CI = 0.61, 0.75).

In addition, Levy et al. (2017) retrospectively generated a cohort of smokers who were using traditional cigarettes one year prior to their survey. This research focused on the association between e-cigarette use and having made a quit attempt in the past year, and having been abstinent from traditional cigarettes for a minimum of 12 weeks after a quit attempt. By using multiple logistic regression analysis, their findings suggested that: quit attempt in the past year was related to previous or current e-cigarettes usage and that the probability of smoking cessation for 12 weeks or more was significantly associated with e-cigarette use.

Finally, in terms of the most recent studies, Zhu et al. (2017) examined data from the large U.S. Current Population SurveyTobacco Use Supplement (CPS-TUS). The researchers drew on survey data from more than 160,000 respondents to generate a retrospective cohort of participants who reported having been smokers of traditional cigarettes one year prior to the survey. They examined the population-level rates of making a quit attempt in the past year and of quitting smoking. Smokers who used e-cigarettes during 2014-15 were more likely than non-users to make a quit attempt and obtain complete smoking cessation. The analysis verified that the quit rates between smokers of traditional cigarettes who used or had ever used an e-cigarette showed a smoking cessation rate increased by 1.1 percentage points between 2010-2011 and 2014-2015. This happened in coincidence with the increase of e-cigarette use amongst smokers of traditional cigarettes. Hence, in this study e-cigarettes appear to have helped to improve smoking cessation rates.

All three of these more recent studies have significant limitations. They are observational in nature and thus cannot determine 
causality. In addition, there was likely to be recall bias amongst participants in relation to both the extent of e-cigarette use and quit attempts. Finally, there may be confounders not assessed in the studies, including lack of information on previous quit attempts, the type of e-cigarette used, motivation to quit and reasons for ecigarette use, for example.

In a very recent fourth study, e-cigarettes usage was almost twice as effective as NRT to help smokers of traditional cigarettes quit smoking (Hajek et al., 2019). This multicentre randomised trial of e-cigarettes versus NRT involved 886 adults attending smoking cessation services in England. The smokers were randomised with 447 individuals assigned to a NRT group and 439 assigned to an e-cigarette group. Both groups received one-on-one behavioural counselling each week for four weeks and were biochemically tested at the end of the year to assure they had stopped smoking. Smokers in the e-cigarette group were given a starter pack, a $30 \mathrm{~mL}$ bottle of tobacco flavoured e-liquid with a nicotine concentration of $18 \mathrm{mg}$ per $\mathrm{mL}$, and instructions on how to use the device. Participants were encouraged to use different flavoured eliquids with different concentrations of nicotine. Participants in the NRT group were offered a several products including patches, gum, lozenges, nasal or mouth sprays, inhalers, mouth strips, or microtabs and they were encouraged to choose a combination of products.

The primary outcome was sustained smoking abstinence for one year, which was biochemically validated during the final visit of the trial. Secondary outcomes included participant-reported treatment usage and respiratory symptoms. The researchers observed a one-year abstinence rate of $18.0 \%$ in the e-cigarette group compared with $9.9 \%$ in the NRT group. This study found that $80 \%$ of participants in the e-cigarette group were more likely to continue using their product at one year compared with $9.0 \%$ in the NRT group. This study had several strong points, including long-term outcomes with a large sample size, a real life setting, researchers who let participants choose their e-liquid and their NRT, biochemical verification of smoking cessation outcomes, and rigorous data analysis but it's uncertain if the results would generalize to other populations of smokers. It is also not possible to affirm that these discoveries would apply to other e-cigarette devices, or away from the setting of a controlled study and lacking behavioural counselling.

\section{Conclusions}

Although e-cigarettes have not been proven to be totally safe, evidence suggests that they may be less harmful alternatives to combustible cigarette smoking. The findings from these studies, conducted with first generation and second-generation ecigarettes, suggests that the provision of e-cigarettes can significantly reduce traditional cigarette consumption and $\mathrm{CO}$ expired breath without worsening of general state of health and without showing significant and serious Adverse Events (AEs). Consequently, for people who smoke e-cigarettes could be considered among the applicable tools for THR, well-being and health empowerment.

\section{References}

Adriaens, K., Van Gucht, D., Declerck, P., \& Baeyens, F. (2014). Effectiveness of the electronic cigarette: An eight-week Flemish study with six-month follow-up on smoking reduc- tion, craving and experienced benefits and complaints. International Journal of Environmental Research and Public Health, 11(11), 11220-11248.

Alberg, A. J., \& Samet, J. M., (2003). Epidemiology of lung cancer. Chest, 123, 21-49.

Bauld, L., Bell, K., McCullough, L., Richardson, L., \& Greaves, L. (2010). The effectiveness of NHS smoking cessation services: a systematic review. Journal of Public Health, 32(1), 71-82.

Brown, A. \& Xing C. (2015) Patent U.S. Patent 9, 215-895.

Bullen, C., Howe, C., Laugesen, M., McRobbie, H., Parag, V., Willima, J., \& Walker, N. (2013). Electronic cigarettes for smoking cessation: a randomized controlled trial. The Lancet, 382(9905), 1629-1637.

Burns, D. M. (1991). Cigarettes and cigarette smoking. Clinic in chest medicine, 12(4), 631-642.

Cahill, K., Lindson-Hawley, N., \& Thomas, K. H. (2016). Nicotine receptor partial agonists for smoking cessation. The Cochrane database of systematic reviews, 18(4).

Cahill, K., Stevens, S., Perera, R., \& Lancaster, T. (2013). Pharmacological interventions for smoking cessation: an overview and network meta-analysis. The Cochrane database of systematic reviews, 31(5).

Campagna, D., Cibella, F., Caponnetto, P., Amaradio, M. D., Caruso, M., Morjaria, J. B., Malerba, M., \& Polosa, R. (2016). Changes in breathomics from a 1-year randomized smoking cessation trial of electronic cigarettes. European Journal of Clinical Investigation, 46(8), 698-706.

Caponnetto, P., Campagna, D., Cibella, F., Morjaria, J. B., Caruso, M., Russo, C., et al. (2013). Correction: EffiCiency and Safety of an eLectroniccigAreTte (ECLAT) as Tobacco Cigarettes Substitute: A Prospective 12-Month Randomized Control Design Study. PLoS ONE 9(1).

Caponnetto, P., Keller, E., Bruno, C. M., \& Polosa, R. (2013). Handling relapse in smoking cessation: strategies and recommendations. Internal and Emergency Medicine, 8(1), 7-12.

Caponnetto, P., Russo, C., Bruno, C. M., Alamo, A., Amaradio, M. D., \& Polosa, R. (2013). Electronic cigarette: a possible substitute for cigarette dependence. Monaldi Archives for Chest Disease: archivio Monaldi per le malattie del torace, 79(1), 12-19.

Centers for Disease Control and Prevention (2004). The health consequences of smoking: a report of the surgeon general. US Department of Health and Human Services: Atlanta, GA: Office of the Surgeon General (US), Office on Smoking and Health (US).

Chen, R., Aherrera, A., Isichei, C., Olmedo, P., Jarmul, S., Cohen, J. E., Navas-Acien, A., \& Rule, A. M. (2017). Assessment of indoor air quality at an electronic cigarette (vaping) convention. Journal of Exposure Science and Environmental Epidemiology, 28, 522-529.

Cibella, F., Campagna, D., Caponnetto, P., Amaradio, M. D., Caruso, M., Russo, C., Cockcroft, D. W., \& Polosa, R. (2016). Lung function and respiratory symptoms in a randomized smoking cessation trial of electronic cigarettes. Clinical Science, 130(21), 1929-1937.

Cobb, N. K., Byron, M. J., Abrams, D. B., \& Shields, P. G. (2010). Novel nicotine delivery systems and public health: The rise of the "e-cigarette". American Journal of Public Health, 100, 2340-2342.

Cooke, W. H., Pokhrel A., Dowling, C., Fogt, D. L., \& Rickards, C. A. (2015). Acute inhalationof vaporized nicotine increases arterial pressure in young non-smokers: A pilot study. Clinical Autonomic Research, 25(4), 267-270. 
Cravo, A. S., Bush, J., Sharma, G., Savioz, R., Martin, C., Craige, S., \& Walele, T. (2016). A randomised, parallel group study to evaluate the safety profile of an electronic vapour product over 12 weeks. Regulatory Toxicology and Pharmacology, 81, S1S14.

D'ruiz, C. D., O'Connell, G., Graff, D. W., \& Yan, X. S. (2017). Measurement of cardiovascular and pulmonary function endpoints and other physiological effects following partial or complete substitution of cigarettes with electronic cigarettes in adult smokers. Regulatory Toxicology and Pharmacology, 87, 36-53.

Dawkins, L., \& Corcoran, O. (2014). Acute electronic cigarette use: Nicotine delivery and subjective effects in regular users. Psychopharmacology, 231(2), 401-407.

Dawkins, L., Munafo, M., Christoforou, G., Olumegbon, N., \& Soar, K. (2016). The effects of e-cigarette visual appearance on craving and withdrawal symptoms in abstinent smokers. Psychology of addictive behaviors, 30(1), 101-105.

de Marco, R., Accordini, S., Cerveri, I., Corsico, A., Sunyer, J., Neukirch, F., Künzli, N., Leynaert, B., Janson, C., Gislason, T., Vermeire, P., Svanes, C., Anto, J. M., Burney, P., \& European Community Respiratory Health Survey Study Group (2004). An international survey of chronic obstructive pulmonary disease in young adults according to GOLD stages. Thorax, 59(2), 120-125.

DeMeo, D. L., Carey, V. J., Chapman, H. A., Reilly, J. J., Ginns, L. C., Speizer, F. E., Weiss, S. T., \& Silverman, E. K. (2004). Familial aggregation of FEF25-75 and FEF25-75/FVC in families with severe, early onset COPD. Thorax, 59(5), 396400.

DiFranza, J. R., \& Lew, R. A. (1996). Morbidity and mortality in children associated with use of tobacco products by other people. Pediatrics, 97(4), 560-568.

Doll, R., Peto, R., Boreham, J., \& Sutherland, I. (2004). Mortality in relation to smoking: 50 years' observations on male British doctors. BMJ, 328(7455), 1519-1528.

El Dib, R., Suzumura, E. A., Akl, E. A., Gomaa, H., Agarwal, A., Chang, Y., Prasad, M., Ashoorion, V., Heels-Ansdell, D., Maziak, W., \& Guyatt, G. (2017). Electronic nicotine delivery systems and/or electronic non-nicotine delivery systems for tobacco smoking cessation or reduction: A systematic review and meta-analysis. BMJ open, 7(2), e012680.

Etter, J. F. (2010). Electronic cigarettes: a survey of users. BMC public health, 10(1), 231.

Etter, J.F, \& Bullen, C. (2011). Electronic cigarette: users profile, utilization, satisfaction and perceived efficacy. Addiction, 106, 2017-2028.

Ezzati, M., \& Lopez, A. D. (2003). Estimates of global mortality attributable to smoking in 2000. The Lancet, 362(9387), 847852.

Faner, R., Tal-Singer, R., Riley, J. H., Celli, B., Vestbo, J., MacNee, W., Bakke, P., Calverley, P. M., Coxson, H., Crim, C., Edwards, L. D., Locantore, N., Lomas, D. A., Miller, B. E., Rennard, S. I., Wouters, E. F., Yates, J. C., Silverman, E. K., Agusti, A., \& ECLIPSE Study Investigators (2014). Lessons from ECLIPSE: A review of COPD biomarkers. Thorax, 69(7), 666-672.

Farsalinos, K. \& Polosa, R. (2014). Safety evaluation and risk assessment of electronic cigarettes as tobacco cigarette substitutes: a systematic review. Therapeutic Advances in Drug Safety, 5(2), 67-86.

Farsalinos, K. E., Spyrou, A., Tsimopoulou, K., Stefopoulos, C., Romagna, G., \& Voudris V. (2014). Nicotine absorption from electronic cigarette use: comparison between first and newgeneration devices. Scientific Reports, 4, 4133.

Flanders, W. D., Lally, C. A., Zhu, B. P., Henley, S. J., \& Thun, M. J. (2003). Lung cancer mortality in relation to age, duration of smoking, and daily cigarette consumption: results from Cancer Prevention Study II. Cancer Research, 63(19), 6556-6562.

Fogt, D. L., Levi, M. A., Rickards, C. A., Stelly, S. P., \& Cooke, W. H. (2016). Effects of acute vaporized nicotine in non-tobacco users at rest and during exercise. International Journal of Exercise Science, 9(5), 607-615.

Franco, T., Trapasso, S., Puzzo, L., \& Allegra, E. (2016). Electronic cigarette: Role in the primary prevention of oral cavity cancer. Clinical Medicine Insights: Ear, Nose and Throat. 9, 7-12.

Gallus, S., Lugo, A., Colombo, P., Pacifici, R., La Vecchia, C. (2013). Smoking prevalence in Italy 2011 and 2012, with a focus on hand-rolled cigarettes. Preventive Medicine, 56: 314318.

Gallus, S., Zuccaro, P., Colombo, P., Apolone, G., Pacifici, R., Garattini, S., La Vecchia, C. (2006). Effects of new smoking regulations in Italy. Annals of Oncology, 17: 346-347.

Gilbert, H. A. (1965). Smokeless non-tobacco cigarette $(3,200.819)$. Retrieved from http://www.njgasp.org/ patents/US3200819.

Giovenco, D. P., \& Delnevo, C. D. (2018). Prevalence of population smoking cessation by electronic cigarette use status in a national sample of recent smokers. Addictive behaviors, 76, 129-134.

Glasser, A. M., Collins, L., Pearson, J. L., Abudayyeh, H., Niaura, R. S., Abrams, D. B., \& Villanti, A. C. (2017). Overview of electronic nicotine delivery systems: a systematic review. American Journal of Preventive Medicine, 52(2), e33e66.

Goldenson, N. I., Kirkpatrick, M. G., Barrington-Trimis, J. L., Pang, R. D., McBeth, J. F., Pentz, M. A., Samet, J. M., \& Leventhal, A. M. (2016). Effects of sweet flavorings and nicotine on the appeal and sensory properties of e-cigarettes among young adult vapers: Application of a novel methodology. Drug and Alcohol Dependence, 168, 176-180.

Goniewicz, M. L., Smith, D. M., Edwards, K. C., Blount, B. C., Caldwell, K. L., Feng, J., Wang, L., Christensen, C., Ambrose, B., Borek, N., van Bemmel, D., Konkel, K., Erives, G., Stanton, C. A., Lambert, E., Kimmel, H. L., Hatsukami, D., Hecht, S. S., Niaura, R. S., Travers, M., Lawrence, C., \& Hyland, A. J. (2018). Comparison of Nicotine and Toxicant Exposure in Users of Electronic Cigarettes and Combustible Cigarettes. JAMA Network Open, 1(8):e18593

Hartmann-Boyce J, McRobbie H, Bullen C, Begh R, Stead LF, \& Hajek P. (2016) Can electronic cigarettes help people stop smoking, and are they safe to use for this purpose? Cochrane Database of Systematic Reviews, 9.

Hon, L. (2003). U.S. Patent 8393331 B2 Electronic Atomization Cigarette, https://docs.google.com/viewer?url= patentimages.storage.googleapis.com/pdfs/US8393331.

Hughes, J.R., Stead, L.F., \& Lancaster, T. (2007). Antidepressants for smoking cessation. Cochrane database of systematic reviews (1):CD000031.

Istituto Nazionale di Statistica (ISTAT) (2015). Annuario statistico Italiano 2013. Available from: https://www.istat.it/it/files//2013/12/ASI_2013.pdf. Accessed December 2, 2017.

Jiménez-Ruiz CA, Masa F, Miravitlles M, Gabriel R, Viejo JL, Villasante C, \& Sobradillo V. (2001). Smoking characteristics: 
Differences in attitudes and dependence between healthy smokers and smokers with COPD. Chest, 119(5), 1365-1370.

Joossens, L., \& Raw, M. (2014). The tobacco control scale 2013 in Europe. A Report pf the Association of European Cancer Leagues. Available from: https://www.cancer.be/sites/default/files/TC-2013-inEurope.pdf. Accessed September 22, 2017.

JUUL. (2018). Manufacturing quality: what is JUUL? San Francisco, CA: JUUL Labs, Inc. https://support.juulvapor.com/home/learn/faqs/manufacturingquality\#materials. (Accessed June 14, 2018).

Kalkhoran, S., \& Glantz, S. A. (2016). E-cigarettes and smoking cessation in real-world and clinical settings: a systematic review and meta-analysis. The Lancet Respiratory Medicine, 4(2), 116-128.

Kee, C. (2018). Everything you need to know about the JUUL, including the health effects: Buzzfeed News, 2018.

Khoudigian, S., Devji, T., Lytvyn, L., Campbell, K., Hopkins, R., \& O'reilly, D. (2016). The efficacy and short-term effects of electronic cigarettes as a method for smoking cessation: A systematic review and a meta-analysis. International Journal of Public Health, 61(2), 257-267.

La Vecchia, C., Franceschi, S., \& Levi, F. (2003). Epidemiological research on cancer with a focus on Europe. European Journal of Cancer Prevention, 12(1), 5-14.

Lawler R (2018). Vaporization startup Pax Labs introduces Juul, its next-gen e-cigarette. Techcrunch.com 2015. https://techcrunch.com/2015/04/21/pax-juul/. Accessed June 14, 2018.

Lee, Y. O., \& Kim, A. E. (2015). 'Vape shops' and 'E-Cigarette lounges' open across the USA to promote ENDS. Tobacco Control, 24(4), 410-412.

Levy, D. T., Yuan, Z., Luo, Y., \& Abrams, D. B. (2017). The relationship of E-cigarette use to cigarette quit attempts and cessation: insights from a large, nationally representative US survey. Nicotine \& Tobacco Research, 1-9.

Ling, P. M., \& Glantz, S. A. (2005). Tobacco industry consumer research on socially acceptable cigarettes. Tobacco Control, 14(5), e3-e3.

Lugo, A., Asciutto, R., Pacifici, R., Colombo, P., La Vecchia, C., \& Gallus, S. (2015). Smoking in Italy 2013-2014, with a focus on the young. Tumori Journal, 101(5), 529-534.

Lundbäck, B., Lindberg, A., Lindström, M., Rönmark, E., Jonsson, A. C., Jönsson, E., Larsson, L. G., Andersson, S., Sandström, T., Larsson, K., \& Obstructive Lung Disease in Northern Sweden Studies (2003). Not 15 but $50 \%$ of smokers develop COPD? Report from the Obstructive Lung Disease in Northern Sweden studies. Respiratory Medicine, 97(2), 115-122.

Manzoli, L., Flacco, M. E., Ferrante, M., La Vecchia, C., Siliquini, R., Ricciardi, W. (2017). Cohort study of electronic cigarette use: effectiveness and safety at 24 months. Tobacco Control, 26(3), 284-292.

Moheimani, R. S., Bhetraratana, M., Yin, F., Peters, K. M., Gornbein, J., Araujo, J. A., \& Middlekauff, H. R. (2017). increased cardiac sympathetic activity and oxidative stress in habitual electronic cigarette users: implications for cardiovascular risk. JAMA cardiology, 2(3), 278-284.

Mooney, M. E., \& Sofuoglu, M. (2006). Bupropion for the treatment of nicotine withdrawal and craving. Expert Review of Neurotherapeutics, 6(7), 965-81.

National Institute for Health and Care Excellence [NICE]. (2011). Public health guidance scope: Tobacco: Harm-reduction approaches to smoking. London: NICE.
NICE. (2013). Public Health Guideline 45Tobacco: Harm-reduction approaches to smoking. London: NICE.

Osservatorio Fumo, Droga e Alcol (OSSFAD). (2016). Osservatorio Droga Alcol e Fumo. Rapporto annuale sul fumo. OSSFAD. (2018). Rapporto annuale sul fumo.

Perfetti, T. A., \& Rodgman, A. (2013). The chemical components of tobacco and tobacco smoke. New York: NY CRC press.

PHS Guideline Update Panel, Liaisons, and Staff (2008). Treating tobacco use and dependence: 2008 update, US Department of Health and Human Services, Public Health Service. Respiratory care, 53(9), 1217-22.

Polosa, R., Cibella, F., Caponnetto, P., Maglia, M., Prosperini, U., Russo, C., Tashkin, D. (2017). Health impact of E-cigarettes: a prospective 3.5-year study of regular daily users who have never smoked. Scientific Reports, 7(1), 13825.

Polosa, R., Morjaria, J. B., Caponnetto, P., Campagna, D., Russo, C., Alamo, A., et al. (2014a). Effectiveness and tolerability of electronic cigarette in real-life: a 24-month prospective observational study. Internal and Emergency Medicine, 9(5), 537546.

Polosa, R., Morjaria, J. B., Caponnetto, P., Caruso, M., Campagna, D., Amaradio, M. D., Ciampi, G., Russo, C., \& Fisichella, A. (2016). Persisting long term benefits of smoking abstinence and reduction in asthmatic smokers who have switched to electronic cigarettes. Discovery medicine, 21(114), 99-108.

Polosa R, Morjaria JB, Caponnetto P, Prosperini U, Russo C, Pennisi A, Bruno CM. (2016). Evidence for harm reduction in COPD smokerswho switch to electronic cigarettes. Respiratory research, 17(1), 166.

Polosa, R., Morjaria, J., Caponnetto, P., Caruso, M., Strano, S., Battaglia, E., \& Russo, C. (2014). Effect of smoking abstinence and reduction in asthmatic smokers switching to electronic cigarettes: evidence for harm reversal. International Journal of Environmental Research and Public Health, 11(5), 4965-4977.

Public Health England [PHE] (2016). E-cigarettes: a developing public health consensus. https://assets.publishing.service.gov. uk/government/uploads/system/uploads/attachment_data/file/ 534708/E-cigarettes_joint_consensus_statement_2016.pdf

Pulvers, K., Emami, A. S., Nollen, N. L., Romero, D. R., Strong, D. R., Benowitz, N. L., \& Ahluwalia, J. S. (2016). Tobacco consumption and toxicant exposure of cigarette smokers using electronic cigarettes. Nicotine and Tobacco Research, 20(2), 206-214.

Radding B (2015). Pax Juul: the iPhone of e-cigs? Mens Fit 2015

Richtel, M. A. T. T. (2014). Selling a poison by the barrel: liquid nicotine for e-cigarettes. New York Times, 23.

Russell, M. A. H. (1974). Realistic goals for smoking and health: A case for safer smoking. The Lancet, 1, 254-258

Sanford, Z., Goebel, M. D., \& Lynne, J. (2014). E-cigarettes: an up to date review and discussion of the controversy. West Virginia Medical Journal, 110(4), 10-5.

Shahab, L., Goniewicz, M. L., Blount, B. C., Brown, J., McNeill, A., Alwis, K. U., Feng, J., Wang, L., \& West, R. (2017). Nicotine, carcinogen, and toxin exposure in long-term e-cigarette and nicotine replacement therapy users: a cross-sectional study. Annals of Internal Medicine, 166(6), 390-400.

Siegel, R. L., Jacobs, E. J., Newton, C. C., Feskanich, D., Freedman, N. D., Prentice, R. L., \& Jemal, A. (2015). Deaths due to cigarette smoking for 12 smoking-relatedcancers in the United States. JAMA Internal Medicine, 175(9), 1574-1576.

Sleight, V. J. (2016). A brief history of the electronic cigarette. Journal of Lung, Pulmunary \& Respiratory Research 3(5), 12. 
Spindle, T. R., Hiler, M. M., Breland, A. B., Karaoghlanian, N. V., Shihadeh, A. L., \& Eissenberg, T. (2016). The influence of a mouthpiece-based topography measurement device on electronic cigarette user's plasma nicotine concentration, heart rate, and subjective effects under directed and ad libitum use conditions. Nicotine \& Tobacco Research, 19(4), 469-476.

St. Helen, G. S., Dempsey, D. A., Havel, C. M., Jacob III, P., \& Benowitz, N. L. (2017). Impact of e-liquid flavors on nicotine intake and pharmacology of e-cigarettes. Drug and Alcohol Dependence, 178, 391-398.

Stead, L. F., Perera, R., Bullen, C., Mant, D., Hartmann-Boyce, J., Cahill, K., \& Lancaster, T. (2012) Nicotine replacement therapy for smoking cessation. The Cochrane Database of Systematic Reviews, CD000146.

Stephens, W. E. (2018). Comparing the cancer potencies of emissions from vapourised nicotine products including e-cigarettes with those of tobacco smoke. Tobacco Control, 27(1), 10.

Stringhini, S., Carmeli, C., Jokela, M., Avendaño, M., Muennig, P., Guida, F., Ricceri, F., d'Errico, A., Barros, H., Bochud, M., Chadeau-Hyam, M., Clavel-Chapelon, F., Costa, G., Delpierre, C., Fraga, S., Goldberg, M., Giles, G. G., Krogh, V., KellyIrving, M., Layte, R., Lasserre, A.M., Marmot, M.G., Preisig, M., Shipley, M.J., Vollenweider, P., Zins, M., Kawachi, I., Steptoe, A., Mackenbach, J.P., Vineis, P., Kivimäki, M.; LIFEPATH consortium. (2017). Socioeconomic status and the $25 \times 25$ risk factors as determinants of premature mortality: a multicohort study and meta-analysis of 17 million men and women. The Lancet, 389 (10075), 1229-1237.

Strongin R. M. (2019). E-Cigarette Chemistry and Analytical Detection. Annual Review of Analytical Chemistry (Palo Alto, Calif.), 12(1), 23-39.

Szołtysek-Bołdys, I., Sobczak, A., Zielińska-Danch, W., Bartoń, A., Koszowski, B., \& Kośmider, L. (2014). Influence of inhaled nicotine source on arterialstiffness. Przegladlekarski, 71(11), 572-575.

Tobacco Atlas. The Tobacco Atlas. 5th ed. Atlanta, GA: American Cancer Society; 2015. Available from: http:// www.tobaccoatlas.org/. Accessed June 18, 2018.

Tramacere, I., Gallus, S., Fernandez, E., Zuccaro, P., Colombo, P., \& La Vecchia, C. (2009). Medium-term effects of the Italian smoke-free legislation: findings from four annual populationbased surveys. Journal of Epidemiology \& Community Health, 63(7), 559-562.

Truth Initiative. What is JUUL? Washington, DC: Truth Initiative, 2018. Accessed June 13, 2018.

Vansickel, A. R., Cobb, C. O., Weaver, M. F., \& Eissenberg, T. E. (2010). A clinical laboratory model for evaluating the acute effects of electronic "cigarettes": nicotine delivery profile and cardiovascular and subjective effects. Cancer Epidemiology and Biomarkers \& Prevention, 19(8), 1945-1953.

Viegi, G., Pedreschi, M., Baldacci, S., Chiaffi, L., Pistelli, F.,
Modena, P., Vellutini, M., Di Pede, F., \& Carrozzi, L. (1999). Prevalence rates of respiratory symptoms and diseases in general population samples of North and Central Italy. The International Journal of Tuberculosis and Lung Disease, 3(11), 1034-1042.

Wedzicha, J. A., \& Donaldson, G. C. (2003). Exacerbations of chronic obstructive pulmonary disease. Respiratory Care, 48(12), 1204-1215.

World Health Organization (WHO) (2003): WHO Framework Convention on Tobacco Control. Geneva: WHO Press.

World Health Organization (WHO) (2008). World Health Statistics 2008. Geneva: WHO. Available from: https://www.who.i nt/whosis/whostat/2008/en/. (Accessed April 1, 2018).

World Health Organization (WHO) (2012). WHO global report: Mortality attributable to tobacco. Geneva: WHO. Available from: https:/www.who.int/tobacco/ publications/surveillance/rep_mortality_attributable/en/. Accessed January 1, 2016.

World Health Organization (WHO) (2013). WHO report on the global tobacco epidemic, 2013. Enforcing bans on tobacco advertising, promotion and sponsors. Geneva: WHO. Available from: https://apps.who.int/iris/bitstream/handle/ 10665/85380/9789241505871 eng.pdf;jsessionid=4F65AB79 2407D94DCA7381BF89EC3D44? sequence $=1$

World Health Organization (WHO) (2017). World No Tobacco Day 2017: Beating tobacco for health, prosperity, the environment and national development. Geneva: WHO. http://www.who.int/mediacentre/news/releases/2017/notobacco-day/en/. Accessed 25, November 2017.

World Health Organization (WHO) (2018). WHO global report: Mortality attributable to tobacco. Geneva: WHO. Available form: http://www.who.int/gho/database/en/. Accessed February 11, 2018.

Yan, X. S., \& D'Ruiz, C. (2015). Effects of using electronic cigarettes on nicotine delivery and cardiovascular function in comparison with regular cigarettes. Regulatory Toxicology and Pharmacology, 71(1), 24-34.

Zemann A. (2011) Smoke chemistry. In: Bernhard D, ed. Cigarette Smoke Toxicity. Weinheim: Wiley-VCH Verlag \& Co. KGaA; pp. 55-64.

Zenzes, M. T. (2000). Smoking and reproduction: gene damage to human gametes and embryos. Human Reproduction Update, 6 (2), 122-131

Zhu, S. H., Sun, J. Y., Bonnevie, E., Cummins, S. E., Gamst, A., Yin, L., \& Lee, M. (2014). Fourhundred and sixty brands of ecigarettes and counting: implications for product regulation. Tobacco Control, 23(3), 3-9.

Zhu, S. H., Zhuang, Y. L., Wong, S., Cummins, S. E., \& Tedeschi, G. J. (2017). E-cigarette use and associated changes in population smoking cessation: evidence from US current population surveys. The BMJ, 358, j3262. 